\title{
Nightmare Frequency, Nightmare Distress and the Efficiency of Trauma-Focused Cognitive Behavioral Therapy for Post-Traumatic Stress Disorder
}

\author{
Katia Levrier, ${ }^{1}$ Andre Marchand, ${ }^{1}$ Genevieve Belleville, ${ }^{2,}{ }^{*}$ Beaulieu-Prevost Dominic, ${ }^{3}$ and Stephane \\ Guay $^{4}$ \\ ${ }^{1}$ Department of Psychology, University of Quebec a Montreal, Quebec, Canada \\ ${ }^{2}$ Department of Psychology, School of Psychology, University of Laval, Quebec, Canada \\ ${ }^{3}$ Department of Sexology, University of Quebec a Montreal, Quebec, Canada \\ ${ }^{4}$ School of Criminology, University of Montreal, Quebec, Canada \\ "Corresponding author: Genevieve Belleville, Department of Psychology, School of Psychology, University of Laval, Bibliotheques st., Quebec, Canada. Tel: +1-4186562131, E-mail: \\ genevieve.belleville@psy.ulaval.ca
}

Received 2015 September 22; Revised 2016 January 07; Accepted 2016 January 25.

\begin{abstract}
Background: Up to $71 \%$ of trauma victims diagnosed with PTSD have frequent nightmares (NM), compared to only $2 \%$ to $5 \%$ of the general population.

Objectives: The present study examined whether nightmares before the beginning of cognitive behavior therapy (CBT) for posttraumatic stress disorder (PTSD) could influence overall PTSD symptom reduction for 71 individuals with PTSD and different types of traumatic events.

Patients and Methods: Participants received a validated CBT of 20 weekly individual sessions. They were evaluated at five measurement times: at pre-treatment, after the third and ninth session, at post-treatment, and at 6 months follow-up.

Results: The presence of nightmares did not impact overall CBT efficiency. Specific CBT components were efficient in reducing the frequency and distress of nightmares.

Conclusions: Most participants no longer had PTSD but some still had nightmares.
\end{abstract}

Keywords: PTSD, Dream, Cognitive-Behavior Therapy, Trauma, Sleep, Nightmare

\section{Background}

Up to $71 \%$ of trauma victims diagnosed with PTSD have frequent nightmares (NM), compared to only $2 \%$ to $5 \%$ of the general population (1). Empirical findings showed that NM before a traumatic event could contribute to the development and the maintenance of PTSD symptoms (1), that they were associated with depression (2), that they reduced sleep quality (3), and that they were not specific to one type of trauma, although they were often reported by combat veterans and victims of sexual assault (4).

To date, the treatment recommended by the international society for traumatic stress studies for overall PTSD symptoms is trauma-focused cognitive behavioral therapy (CBT) $(5,6)$. According to the rationale of trauma-focused cognitive behavioral therapy (CBT), traumatic events can contribute to create a memory network called the fear structure and the dysfunctional connections of that structure can be modified through imaginal or in vivo exposure
(5). According to this model, NM are considered to be one element of the fear structure and are thus perceived as a symptom of re-experience that simply occurs at night. If that was the case, NM should no longer be present when PTSD is successfully treated. However, some studies have indicated that NM persisted in a clinically significant manner after CBT (e.g. $(7,8))$.

Studies have generally examined the global impact of CBT as a whole on NM reduction. However, CBT is a multicomponent treatment and the effect of its various strategies on sleep can vary. A study from Salcioglu et al. (9) tested the efficacy of in vivo exposure with 59 survivors of an earthquake. Although NM were significantly reduced after this intervention, they remained a clinically significant symptom. In a more recent study (7), the efficacy of cognitive processing therapy (CPT) and prolonged exposure (PE) were compared among female rape victims with PTSD. Both CPT and PE had large effects on NM reduction, but neither of them achieved complete NM remission. 
Studies reporting NM before and after CBT in a traumatic context are rare (10) and studies monitoring the impact of individual CBT strategies for PTSD on NM using validated instruments are even more limited. No study has investigated the impact of NM on CBT for PTSD efficacy. Such research would help clarify the potential role of NM in PTSD remission and assess the need to include a specific NM intervention in PTSD treatment.

\section{Objectives}

The objectives were to determine whether the presence of NM before the beginning of CBT for PTSD influences PTSD symptom reduction and to examine the efficacy of each CBT strategy on NM reduction.

\section{Patients and Methods}

This study's prospective, longitudinal and repeated measures design (11) was conducted on participants referred to the trauma studies centre of the institut universitaire en sante mentale de montreal (IUSMM) in Canada. The ethics committee of the IUSMM approved this research project. All participants $(n=71)$ signed an informed consent. Inclusion criteria were: a) to be aged between 18 and 65 years old; b) to have been exposed to at least one traumatic event in adulthood; c) to present PTSD as a primary diagnosis according to the DSM-IV-TR (12). The exclusion criteria were: a) repeated war-related traumas; b) alcohol or substance abuse/dependence; c) active suicidal ideations, d) past or present psychotic episode, e) past or present psychotic episode, bipolar disorder, or organic mental disorder, f) ongoing threat (e.g., continued harassment) and g) overwhelming personal problems (e.g., attendance to a trial); and h) severe personality disorder. Participants were asked to refrain from adjusting their psychotropic medication and to refrain from engaging in an additional psychotherapy.

Participants were assessed five times: before the beginning of the treatment (pre-treatment), after the psychoeducation and diaphragmatic breathing component (3rd session; 11 ), after the imaginal exposure component (9th session; T2), at the end of the treatment (post-treatment), and at 6-months follow-up.

The empirically validated CBT for PTSD protocol $(5,13)$ consisted of 20 weekly individual sessions of 90 minutes. Sessions one to three were characterized by psychoeducation regarding PTSD symptoms and anxiety management strategies focused on diaphragmatic breathing. Sessions four to nine consisted of practice imaginal exposure to memories of the traumatic event with cognitive restructuring if necessary. Sessions 10 to 20 were in vivo exposure to avoided situations and relapse prevention. Participants received a manual and therapists followed a standardized intervention protocol.

\subsection{Measurement}

Participants completed the structured clinical interview for DSM-IV-TR Axis I disorders (SCID-I) (14). They also completed the life events checklist (LEC; (15)) to screen for potentially traumatic events. PTSD severity was evaluated by the clinician-administered PTSD scale (CAPS) (16). The frequency and distress of the B2 item were retrieved from the CAPS and used to determine NM presence (frequency) and distress. All questionnaires demonstrate good psychometric properties.

\subsection{Statistical Analysis}

Data were analyzed using SPSS v.17.0 software. The five measurement times were included in the analysis. No differences between completers and non-completers were observed on the sociodemographic variables (except for education: $\left.\mathrm{X}^{2}(1,87)=8.17, \mathrm{P}<.05\right)$, and severity of the CAPS symptoms.

Because the CAPS-B2 item (frequency and distress) did not meet the normality hypothesis, it was dichotomized as 0 (=no symptoms or mild symptoms; that is, a score of 0 or 1 on the CAPS) and 1 (= presence of symptoms; which corresponds to a score of 2,3 or 4 ).

Because all the correlations between age, sex and time since the event and the different dependant variables were not significant, they were not included in the analyses. Data analyses were performed using descriptive statistics (frequency, mean and standard deviation for each variable) and inferential statistics (ANOVA, t-test, chi-square, nonparametric Cochran's Q tests, pairwise comparisons using continuity-corrected McNemar's tests with Bonferroni correction). When the assumption of sphericity is violated for the ANOVA, the Greenhouse-Geisser correction will be considered. The significance level was set at 0.05 for each test.

\subsection{Ethical Consideration}

Participants did not receive any financial compensation.

\section{Results}

Perfect (100\%) inter-rater agreements were obtained for the evaluations at pre-test, and $91.3 \%$ at post-test for the PTSD diagnosis. At baseline, inter-rater agreement was computed based on 34\% of the completers and 31\% of the non-completers. At post-treatment, it was calculated based 
on $32 \%$ of the completers. Therapeutic integrity was also good, with $99.6 \%$ agreement.

The recruitment chart is presented in Figure 1. A total of 71 participants completed the treatment, and 5 participants were lost at 6 months follow-up. The attrition rate was $18.4 \%$.

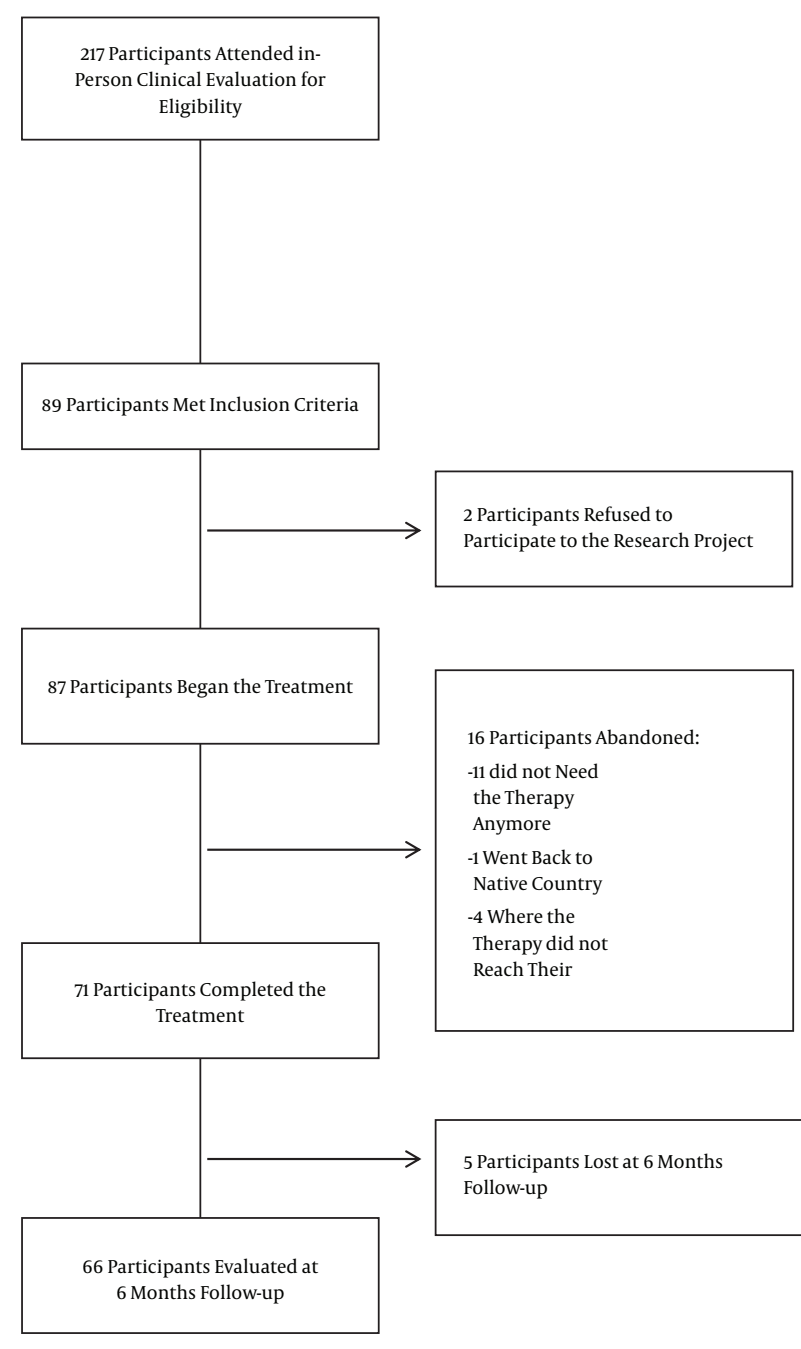

Figure 1. Flowchart of Participents

Most participants (64.8\%) were women, and mean age was 38.65 years $(S D=1.35)$ (see Table 1$)$. Baseline CAPS mean score was 78.58 (SD = 13.08), suggesting high symptom severity. Participants reported four main categories of trauma associated with their current PTSD: physical harm (48\%); accident/catastrophe (31\%); death-related stress (18\%); and others (3\%). The average time interval since trauma was two years $(\mathrm{SD}=3.97)$. Most participants (70.3\%) reported NM related to their traumatic event at pre- treatment.

Table 1. Baseline Characteristics of Completers Sample ${ }^{\mathrm{a}}$

\begin{tabular}{|c|c|c|}
\hline Characteristics & $\mathbf{N}(\%)$ & Mean \pm SD \\
\hline Gender, Female & $46(64.80)$ & \\
\hline Age & & $38.65 \pm 1.35$ \\
\hline \multicolumn{3}{|l|}{ Marital Status } \\
\hline Single & $40(56.30)$ & \\
\hline Married or Common-Law Relationship & $31(43.70)$ & \\
\hline \multicolumn{3}{|l|}{ Employment Status } \\
\hline Employed & $19(26.80)$ & \\
\hline Leave of Absence & $15(21.10)$ & \\
\hline Unemployed & $37(52.10)$ & \\
\hline \multicolumn{3}{|l|}{ Education } \\
\hline Primary & $0(0.00)$ & \\
\hline Secondary & $21(29.60)$ & \\
\hline Collegial & $35(49.30)$ & \\
\hline University & $15(21.10)$ & \\
\hline \multicolumn{3}{|l|}{ Types of Trauma } \\
\hline Accident/Catastrophe & $22(31.00)$ & \\
\hline Physical Harm & $34(47.90)$ & \\
\hline Death-Related Stress & $13(18.30)$ & \\
\hline Other & $2(2.80)$ & \\
\hline Time Interval Since Trauma (in Years) & & $2.23 \pm 3.97$ \\
\hline CAPS Severity & & $78.58 \pm 13.08$ \\
\hline \multicolumn{3}{|l|}{ Comorbid Disorders } \\
\hline Anxiety Disorder & $11(15.50)$ & \\
\hline Mood Disorder & $36(50.70)$ & \\
\hline Alcohol/Substance Abuse Disorder & $3(4.20)$ & \\
\hline
\end{tabular}

Abbreviation: CAPS, Clinician Administered PTSD scale. ${ }^{\mathrm{a}} \mathrm{N}=71$

At post-treatment, 73\% of the participants did not meet PTSD diagnosis criteria. At follow-up, 69\% no longer met PTSD diagnosis and 31\% still met the criteria. CBT had a significant effect on the total score of the CAPS $(\mathrm{F}(2.76,176.57)$ $=94.75, \mathrm{P}<0.001, \mathrm{y}^{2}$ within $\left.=0.60\right)$. The CAPS total score significantly decreased until the end of the treatment, but no statistical difference was observed between the posttreatment and the follow-up. At pre-treatment, its mean was 78.58 (SD =13.08); at T1, 69.13 (SD = 18.36); at T2, 51.97 (SD $=26.50)$; at post-treatment $33.00(\mathrm{SD}=26.85)$; and at followup of 36.68 ( $\mathrm{SD}=30.78)$.

The results of the repeated ANOVA for NM difficulties (without including sleep data) indicated that the presence of $\mathrm{NM}$ (frequency) $[\mathrm{F}(2.77,171.74)=1.59, \mathrm{P}=.20]$ and $\mathrm{NM}$ dis- 
tress $[\mathrm{F}(2.75,170.58)=1.40, \mathrm{P}=.25]$ before the beginning of the CBT had no effect on PTSD symptom reduction.

In Table 2, Cochran analyses show CBT significantly reduced NM frequency and distress $(\mathrm{P}<0.001)$. However, 15 (23.4\%) participants still presented NM (more than twice a week) at post-treatment and $14(21.9 \%)$ at follow-up.

When assessing the impact of each CBT strategy on NM, pairwise comparisons (McNemar tests) revealed a statistically significant decrease for NM frequency between T1 and T2 up to the post-treatment, and with no significant change afterwards (from post-treatment to follow-up). In contrast, for NM distress, we observed a significant decrease from the beginning of the therapy up to T2 and no significant change after the ninth session.

\section{Discussion}

Participants with NM did not benefit less from CBT than those with fewer or no NM. Moreover, CBT had a positive impact on NM reduction with $77 \%$ of participants who did no longer present NM after the treatment. The results demonstrated that CBT had an effect on NM reduction at specific stages in the course of the treatment. First, NM frequency began to decline after practicing imaginal exposure and continued to do so until the end of the therapy, when in vivo exposure was completed. NM distress demonstrated another trajectory with a decrease after psychoeducation and diaphragmatic breathing until the end of the imaginal exposure approach.

The impact of each CBT strategy on NM reduction is interesting as each one impacted distinct aspects of NM. For example, the rationale of diaphragmatic breathing training is to provide a way to reduce patient anxiety when exposed to trauma-related stimuli (5). Available evidence from past studies confirms the efficacy of anxiety management techniques, but results are somewhat mixed in the literature (17-20). In Miller and DiPilato (17), it was demonstrated that progressive deep muscle relaxation training (PDMR) was efficient in reducing the frequency and the intensity of idiopathic NM compared to a wait-list control. Although this previous study was not conducted in a PTSD context, recent studies revealed relationships between high levels of anxiety and NM (18), and thus relaxation training or diaphragmatic breathing in PTSD treatment may help to reduce stress arousal generated during NM. Additionally, Exposure, relaxation, and rescripting treatment (ERRT), which directly addresses NM, uses relaxation and has demonstrated positive results (20). Nevertheless, in a study by Vaughan et al. (19), participants assigned to applied muscle relaxation (AMR) reported no changes in NM frequency.
No studies have tested the impact of psychoeducation on NM distress. As mentioned by Krakow and Zadra (21), individuals seem to remain sceptical on the fact that NM can be treated. Therefore, it could be hypothesized that psychoeducation, during which information is provided to participants on PTSD, could help address this belief and help decrease distress associated with NM.

Finally, the goal of exposure therapy (22), in imagination or in vivo, is to help change the victim's perception of a situation and reaction to this specific and problematic situation by confronting fears. A few studies tried to better understand its impact $(7,9,23)$ and one (9) argued that the efficacy of live exposure on NM (as for other PTSD symptom reduction) might be due to an increased sense of control over fear or distress associated with the trauma.

Although CBT was efficient in reducing NM frequency and distress, NM were still present among $23 \%$ of the participants after the treatment. This result is consistent with earlier findings (7). One explanation for persistent NM after CBT is that NM lead individuals to learn to avoid them. For example, people may adopt avoidance behaviors, such as delayed bed time. This continued behavior can contribute to maintain NM as it is not addressed by CBT (24). Trauma survivors may develop perpetuating factors maintaining NM and thus, NM may need to be specifically addressed in treatment (21). Clinicians have an important role to play in the prevention and early treatment of NM in trauma victims by monitoring them and their content (25). There is still room for improvement for CBT as it relates to NM reduction. Incorporating CBT for NM, such as imagery rehearsal therapy (IRT) is an interesting avenue, as studies have already shown promising results with this approach (26). These results also call for more research in order to identify the most efficient treatment for sleep disturbances in PTSD. For example, therapy sessions may be added if NM are still too frequent or intense. Similarly, it is important to better understand the differential efficacy of treating NM before, during or after CBT for PTSD.

This study had some limitations. The lack of specific NM questionnaires with questions related to distress, such as the nightmare distress questionnaire (NDQ) (27), and weekly sleep diaries would have helped to better understand the sleep course and to differentiate of NM content, i.e. related to the event or not. Furthermore, it may be difficult to generalize our results as NM difficulties were not part of the selection criteria of the study. Participants did not consult for sleep difficulties.

In conclusion, the findings of this research suggest NM do not impact on the CBT for PTSD, and specific therapy components differently impacted NM frequency and distress. Future studies should examine NM, sleep mechanisms and the impact of specific CBT for PTSD strategies on 
Table 2. Prevalences and Cochran Analyses for the Effect of CBT for PTSD on NM Frequency and Distress ${ }^{\mathrm{a}}$

\begin{tabular}{|c|c|c|c|c|c|c|c|}
\hline \multirow[t]{2}{*}{ Variables } & \multirow[t]{2}{*}{ Pre-Treatment } & \multirow[t]{2}{*}{ T1 } & \multirow[t]{2}{*}{ T2 } & \multirow[t]{2}{*}{ Post-Treatment } & \multirow[t]{2}{*}{6 Months Follow-Up } & \multicolumn{2}{|c|}{ Time Effect } \\
\hline & & & & & & $X^{2}(4,64)$ & P Value \\
\hline NM Frequency Presence & $45(70.31)$ & $42(65.63)$ & $28(43.75)$ & $15(23.44)$ & $14(21.88)$ & 60.92 & $<0.001$ \\
\hline NM Distress Presence & $52(81.25)$ & $37(57.81)$ & $23(35.94)$ & $19(29.69)$ & $21(32.81)$ & 56.75 & $<0.001$ \\
\hline
\end{tabular}

Abbreviations: NM, nightmare; PTSD, post-traumatic stress disorder; T1, evaluation after psychoeducation and diaphragmatic breathing component; T2, evaluation after imaginal exposure component.

${ }^{a}$ Values are expressed as No. \%.

these variables.

\section{Acknowledgments}

We would like to thank staff of trauma research center for their cooperation.

\section{Footnotes}

Authors' Contribution: Study design: Andre Marchand, Stephane Guay, Genevieve Belleville and Beaulieu-Prevost Dominic; data collection: Andre Marchand and Stephane Guay; data analysis and interpretation of data: Katia Levrier; drafting of the manuscript: Katia Levrier; critical revision of the manuscript for important intellectual content: Andre Marchand and Genevieve Belleville; study Supervision: Andre Marchand.

Funding/Support: The first author received a doctoral grant from the fonds de recherche en sante du quebec (FRSQ) (Grant No. 21186). The study was founded by the social sciences and humanities research council (SSHRC) (Grant No. 410-2009-1844).

\section{References}

1. van Liempt $S$, van Zuiden $M$, Westenberg $H$, Super A, Vermetten E. Impact of impaired sleep on the development of PTSD symptoms in combat veterans: a prospective longitudinal cohort study. Depress Anxiety. 2013;30(5):469-74. doi: 10.1002/da.22054. [PubMed: 23389990].

2. Pigeon WR, Campbell CE, Possemato K, Ouimette P. Longitudinal relationships of insomnia, nightmares, and PTSD severity in recent combat veterans. J Psychosom Res. 2013;75(6):546-50. doi: 10.1016/j.jpsychores.2013.09.004. [PubMed: 24290044]

3. Davis JL, Byrd P, Rhudy JL, Wright DC. Characteristics of chronic nightmares in a trauma-exposed treatment-seeking sample. Dreaming. $2007 ; \mathbf{1 7}(4): 187-98$.

4. Krakow B, Schrader R, Tandberg D, Hollifield M, Koss MP, Yau CL, et al. Nightmare frequency in sexual assault survivors with PTSD. J Anxiety Disord. 2002;16(2):175-90. [PubMed: 12194543].

5. Foa EB, Keane TM, Friedman MJ, Cohen JA. Effective treatments for PTSD: practice guidelines from the International Society for Traumatic Stress Studies. second ed. New York: Guilford Press; 2008.
6. Shahri SMM, Bahonar E, Nasari M. Trauma-focused cognitive behavioral therapy(TF-CBT) for post-traumatic stress symptoms after traffic accidents. Trauma Monthly. 2015;20(Special Issue).

7. Gutner CA, Casement MD, Stavitsky Gilbert K, Resick PA. Change in sleep symptoms across Cognitive Processing Therapy and Prolonged Exposure: a longitudinal perspective. Behav Res Ther. 2013;51(12):81722. doi: 10.1016/j.brat.2013.09.008. [PubMed: 24184428].

8. Belleville G, Guay S, Marchand A. Persistence of sleep disturbances following cognitive-behavior therapy for posttraumatic stress disorder. I Psychosom Res. 2011;70(4):318-27. doi: 10.1016/j.jpsychores.2010.09.022. [PubMed: 21414451].

9. Salcioglu E, Basoglu M, Livanou M. Effects of live exposure on symptoms of posttraumatic stress disorder: the role of reduced behavioral avoidance in improvement. Behav Res Ther. 2007;45(10):2268-79. doi: 10.1016/j.brat.2007.04.012. [PubMed: 17570342].

10. Belleville G, Cousineau H, Levrier K, St-Pierre-Delorme ME, Marchand A. The impact of cognitive-behavior therapy for anxiety disorders on concomitant sleep disturbances: a meta-analysis. J Anxiety Disord. 2010;24(4):379-86. [PubMed: 20369395].

11. Kazdin AE. Mediators and mechanisms of change in psychotherapy research. Annu Rev Clin Psychol. 2007;3:1-27. doi: 10.1146/annurev.clinpsy.3.022806.091432. [PubMed:17716046].

12. Spitzer RL, Gibbon ME, Skodol AE, Williams JBW, First MB. DSM-IVTR casebook: A learning companion to the diagnostic and statistical manual of mental disorders (text rev.). 4th ed. Washington: American Psychiatric Publishing, Inc.; 2002.

13. Marchand A, Beaulieu-Prevost D, Guay S, Bouchard S, Drouin MS, Germain V. Relative Efficacy of Cognitive-Behavioral Therapy Administered by Videoconference for Posttraumatic Stress Disorder: A SixMonth Follow-Up. J Aggress Maltreat Trauma. 2011;20(3):304-21. doi: 10.1080/10926771.2011.562479. [PubMed: 23687441].

14. Navara CS, First NL, Schatten G. Phenotypic variations among paternal centrosomes expressed within the zygote as disparate microtubule lengths and sperm aster organization: correlations between centrosome activity and developmental success. Proc Natl Acad Sci U S A. 1996;93(11):5384-8. [PubMed: 8643584].

15. Gray MJ, Elhai JD, Owen JR, Monroe R. Psychometric properties of the Trauma Assessment for Adults. Depress Anxiety. 2009;26(2):190-5. doi: 10.1002/da.20535. [PubMed: 19031486].

16. Blake DD, Weathers FW, Nagy LM, Kaloupek DG, Gusman FD, Charney DS, et al. The development of a Clinician-Administered PTSD Scale.J Trauma Stress. 1995;8(1):75-90. [PubMed: 7712061].

17. Miller WR, DiPilato $M$. Treatment of nightmares via relaxation and desensitization: a controlled evaluation. J Consult Clin Psychol. 1983;51(6):870-7. [PubMed: 6140275]

18. Levin R, Nielsen TA. Disturbed dreaming, posttraumatic stress disorder, and affect distress: a review and neurocognitive model. Psychol Bull. 2007;133(3):482-528. doi: 10.1037/0033-2909.133.3.482. [PubMed: 17469988]. 
19. Vaughan K, Armstrong MS, Gold R, O'Connor N, Jenneke W, Tarrier $\mathrm{N}$. A trial of eye movement desensitization compared to image habituation training and applied muscle relaxation in post-traumatic stress disorder.J Behav Ther Exp Psychiatry. 1994;25(4):283-91. [PubMed: 7706505].

20. Davis JL, Wright DC. Exposure, relaxation, and rescripting treatment for trauma-related nighmares. J Trauma Dissociation. 2006;7(1):5-18. doi:10.1300/J229v07n01_02. [PubMed:16618692].

21. Krakow B, Zadra A. Clinical management of chronic nightmares: imagery rehearsal therapy. Behav Sleep Med. 2006;4(1):45-70.

22. Foa EB, Hembree EA, Rothbaum BO. Prolonged exposure therapy for PTSD: processing of traumatic Experiences. New York: Oxford University Press; 2007.

23. Blanchard EB, Hickling EJ, Malta LS, Freidenberg BM, Canna MA, Kuhn $\mathrm{E}$, et al. One- and two-year prospective follow-up of cognitive behavior therapy or supportive psychotherapy. Behav Res Ther. 2004;42(7):74559. doi: 10.1016/S0005-7967(03)00201-8. [PubMed: 15149896].
24. Spoormaker VI, Montgomery P. Disturbed sleep in post-traumatic stress disorder: secondary symptom or core feature?. Sleep Med Rev. 2008;12(3):169-84. doi: 10.1016/j.smrv.2007.08.008. [PubMed 18424196].

25. Rosen C, Adler E, Tiet Q. Presenting concerns of veterans entering treatment for posttraumatic stress disorder. J Trauma Stress. 2013;26(5):640-3. doi: 10.1002/jts.21841. [PubMed: 24123262].

26. Ung VY, Foshaug RR, MacFarlane SM, Churchill TA, Doyle JS, Sydora BC et al. Oral administration of curcumin emulsified in carboxymethyl cellulose has a potent anti-inflammatory effect in the IL-10 genedeficient mouse model of IBD. Dig Dis Sci. 2010;55(5):1272-7. doi: 10.1007/s10620-009-0843-z. [PubMed: 19513843].

27. Na LX, Zhang YL, Li Y, Liu LY, Li R, Kong T, et al. Curcumin improves insulin resistance in skeletal muscle of rats. Nutr Metab Cardiovasc Dis. 2011;21(7):526-33. doi: 10.1016/j.numecd.2009.11.009. [PubMed: 20227862] 